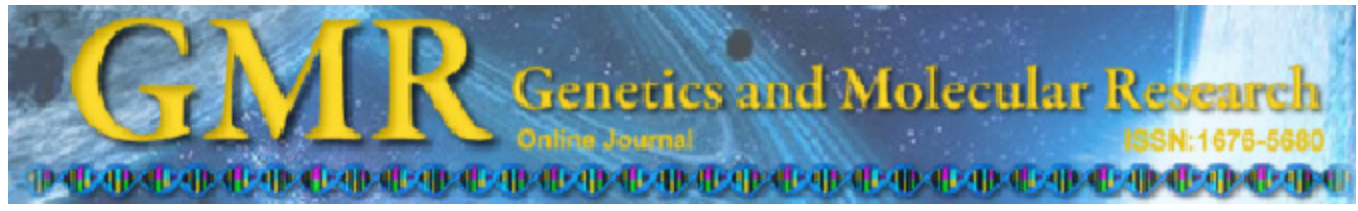

\title{
A case-control study indicates that the $T R I B 1$ gene is associated with pancreatic cancer
}

\author{
X.X. Lu ${ }^{1,2 *}$, J.J. Hu ${ }^{3 *}$, Y. Fang ${ }^{1,2}$, Z.T. Wang ${ }^{4}$, J.J. Xie ${ }^{1}$, Q. Zhan ${ }^{1}$, X.X. Deng ${ }^{1}$, \\ H. Chen ${ }^{1}$, J.B. Jin ${ }^{1}$, C.H. Peng ${ }^{1}$, J. Liu ${ }^{5}$, H.W. Li ${ }^{1}$ and B.Y. Shen ${ }^{1,2}$ \\ ${ }^{1}$ Department of General Surgery, Rui-Jin Hospital, \\ Shanghai Jiaotong University School of Medicine, Shanghai, China \\ ${ }^{2}$ Research Institute of Pancreatic Disease, Rui-Jin Hospital, \\ School of Medicine, Shanghai Jiaotong University, Shanghai, China \\ ${ }^{3}$ Department of Nuclear Medicine, Rui-Jin Hospital, \\ Shanghai Jiaotong University School of Medicine, Shanghai, China \\ ${ }^{4}$ Department of Gastroenterology, Ruijin Hospital, \\ Shanghai Jiaotong University School of Medicine, Shanghai, China \\ ${ }^{5}$ Shanghai Institute of Orthopaedics and Traumatology, Rui-Jin Hospital, \\ Shanghai Jiaotong University School of Medicine, Shanghai, China \\ *These authors contributed equally to this study. \\ Corresponding author: B.Y. Shen \\ E-mail: byshen_rjh@126.com \\ Genet. Mol. Res. 13 (3): 6142-6147 (2014) \\ Received July 31, 2013 \\ Accepted December 3, 2013 \\ Published August 7, 2014 \\ DOI http://dx.doi.org/10.4238/2014.August.7.30
}

ABSTRACT. Pancreatic cancer is a malignant neoplasm originating
from transformed cells arising in tissues that form the pancreas. To
investigate whether the tribbles homolog 1 (Drosophila) gene (TRIB1)
is associated with pancreatic cancer in the Chinese Han population, we
conducted this case-control study and genotyped 3 single nucleotide
polymorphisms (rs 2980879 , rs 2980874 , and rs 2235108 ) of the TRIB1
gene in 182 patients and 359 normal controls of Chinese Han origin
and analyzed their association. The results showed that the rs 2980879 
polymorphism was associated with pancreatic cancer [allele: $\mathrm{P}=$ 0.023434 , genotype: $\mathrm{P}=0.03005$; odds ratio $(\mathrm{OR})$ and $95 \%$ confidence interval $(\mathrm{CI})=0.727788(0.552664-0.958404)]$, whereas the rs2980874 polymorphism had no association with pancreatic cancer [allele: $\mathrm{P}=0.749885$, genotype: $\mathrm{P}=0.699533$; $\mathrm{OR}$ and $95 \% \mathrm{CI}=1.041981$ (0.809196-1.341734)], and the rs2235108 polymorphism was not associated with the disease [allele: $\mathrm{P}=0.629475$, genotype: $\mathrm{P}=$ $0.547534, \mathrm{OR}$ and $95 \% \mathrm{CI}=1.128290(0.690829-1.842770)]$. Haplotype analyses and linkage disequilibrium tests were also conducted, and the results showed that these 3 loci are not in the same block. In conclusion, our study indicated that the TRIBI gene is associated with pancreatic cancer. More studies with larger samples are needed in order to support this finding.

Key words: TRIB1 gene; Pancreatic cancer; Association study; SNPs

\section{INTRODUCTION}

Pancreatic cancer is a malignant neoplasm originating from transformed cells arising in tissues forming the pancreas. It is one of the most common malignant cancers, with a mortality rate virtually equal to its incidence. The main symptoms of pancreatic cancer include abdominal pain, weight loss, diarrhea, and jaundice. However, it is clinically invisible at the early stage of the disease, and most patients are diagnosed at an advanced stage with a very low possibility of therapy (Bardeesy and DePinho, 2002; Li et al., 2004; Siegel et al. 2012). Therefore, it is important to clarify the etiology and to identify the risk factors for the prevention of this lethal disease. Although smoking, diabetes, obesity, history of chronic pancreatitis, and perhaps alcohol drinking are considered as risk factors for pancreatic carcinogenesis (Verma, 2005; Qiu et al., 2005; Lu et al., 2006; Pandey et al., 2011), the detailed etiology of this disease is not currently fully understood. Thus far, the development of pancreatic cancer has been mainly attributed to the combination of environmental and genetic factors (Malats, 2001). In the last decade, a number of genes have been identified to be associated with the susceptibility of pancreatic cancer (Amundadottir et al., 2009; Petersen et al., 2010; Wu et al., 2012).

The tribbles homolog 1 (Drosophila) gene (TRIB1) is located on chromosome 8q24 and encodes a member of the tribbles protein family. It is a serine-threonine kinase-like molecule, which acts as a scaffold protein and plays important roles in mitogen-activated protein kinase (MAPK) signaling cascades (Kiss-Toth et al., 2004; Sung et al., 2007). Studies have revealed that TRIBI is implicated in several diseases such as cancer and myocardial infarction (Kathiresan et al., 2008; Willer et al., 2008; Yokoyama and Nakamura, 2011). However, there is no report about the relationship between TRIB1 and pancreatic cancer, and the prevalence of TRIB1 single nucleotide polymorphisms (SNPs) in this disease remains unclear. To investigate whether the TRIB1 gene is associated with pancreatic cancer in the Chinese Han population, we conducted this case-control study and genotyped 3 SNPs (rs2980879, rs2980874, and rs2235108) of the TRIB1 gene in 182 patients and 359 normal controls of Chinese Han origin and analyzed their association. 


\section{MATERIAL AND METHODS}

\section{Subjects}

This study included 182 patients (100 males and 82 females) diagnosed and histopathologically confirmed with primary pancreatic cancer. They were recruited from the Department of General Surgery of Ruijin Hospital affiliated with Shanghai Jiaotong University School of Medicine. The mean age of the patients was $60.5 \pm 13.1$ years. A total of 359 controls (192 males and 167 females) were randomly selected from healthy individuals who underwent routine health screenings. The mean age of the controls was $52.9 \pm 14.6$ years. The study was approved by the Research Ethics Committee of Ruijin Hospital, Shanghai, China, and informed consent was obtained from all subjects before blood sampling.

\section{Genotyping}

Genomic DNA was isolated from peripheral blood with ethylenediaminetetraacetic acid using the QIAamp blood extraction kit (Qiagen, Hilden, Germany) following manufacturer instructions. The genotyping of TRIB1 SNPs was carried out by the Shanghai Generay Biotech Co., Ltd. (http://www.generay.com.cn/) using allele-specific multiple ligase detection reactions according to standard protocols.

To test the validity of this procedure, about $10 \%$ of samples were confirmed by direct DNA sequencing.

\section{Statistical analysis}

Hardy-Weinberg equilibrium testing in both the healthy control and patient groups, and the allele and genotype frequency analyses were all performed with the SHEsis software (http://analysis.bio-X.cn) (Shi and He, 2005; Liu et al., 2012). All tests were two-tailed and statistical significance was assumed at $\mathrm{P}<0.05$.

\section{RESULTS}

In this case-control study, we genotyped 3 SNPs (rs2980879, rs2980874, and rs2235108) of the TRIB1 gene in 182 patients and 359 normal controls of Chinese Han origin and analyzed their allele frequencies, genotype frequencies, linkage disequilibrium values, and haplotypes. The results showed that the rs 2980879 polymorphism was associated with pancreatic cancer [allele: $\mathrm{P}=0.023434$, genotype: $\mathrm{P}=0.03005$; odds ratio $(\mathrm{OR})$ and $95 \%$ confidence interval $(\mathrm{CI})=0.727788(0.552664-0.958404)]$, whereas the rs 2980874 polymorphism had no association with pancreatic cancer [allele: $\mathrm{P}=0.749885$, genotype: $\mathrm{P}$ $=0.699533 ; \mathrm{OR}$ and $95 \% \mathrm{CI}=1.041981(0.809196-1.341734)]$, and the rs 2235108 polymorphism was not associated with the disease [allele: $\mathrm{P}=0.629475$, genotype: $\mathrm{P}=0.547534$; OR and $95 \% \mathrm{CI}=1.128290(0.690829-1.842770)]$ (Table 1$)$. There was no significant linkage disequilibrium in these 3 SNPs (Table 2). The results of the haplotype analyses showed that these 3 polymorphisms are not in the same block, and there was no significant association among them (Table 3). 
Table 2. Linkage disequilibrium tests.

\begin{tabular}{lcc}
\hline D'value & rs2980874 & rs2235108 \\
\hline rs2980879 & 0.263 & 0.506 \\
rs2980874 & - & 0.100 \\
\hline
\end{tabular}

Table 3. Loci chosen for hap-analysis: rs2980879, rs2980874, and rs2235108.

\begin{tabular}{lrrcccc}
\hline & \multicolumn{1}{c}{ Case (freq) } & Control (freq.) & \multicolumn{1}{c}{$\chi^{2}$} & \multicolumn{1}{c}{ Fisher P } & Perason P & Odds ratio $(95 \% \mathrm{CI})$ \\
\hline A A C & $127.74(0.351)$ & $268.02(0.374)$ & 0.770 & 0.380108 & 0.380062 & $0.887(0.677-1.160)$ \\
A A T & $0.01(0.000)$ & $3.87(0.005)$ & - & - & - & - \\
A G C & $107.88(0.296)$ & $242.16(0.338)$ & 2.281 & 0.131036 & 0.130963 & $0.807(0.611-1.066)$ \\
A G T & $9.38(0.026)$ & $14.95(0.021)$ & - & - & - & - \\
T A C & $33.76(0.093)$ & $42.43(0.059)$ & 3.986 & 0.045946 & 0.045909 & $1.612(1.005-2.586)$ \\
T A T & $10.50(0.029)$ & $16.68(0.023)$ & - & - & - & - \\
T G C & $69.63(0.191)$ & $108.39(0.151)$ & 2.627 & 0.105140 & 0.105074 & $1.318(0.943-1.841)$ \\
T G T & $5.11(0.014)$ & $19.50(0.027)$ & - & - & - & - \\
\hline
\end{tabular}

Global $\chi^{2}$ is 7.789120 while d.f. $=3$ (frequencies $<0.03$ were ignored). Fisher $\mathrm{P}$ value is 0.050697 ; Pearson $\mathrm{P}$ value is 0.050591 .

\section{DISCUSSION}

Tribbles is an evolutionarily conserved protein family that was originally identified in Drosophila. To date, three members of the mammalian tribbles family have been identified, Trib1, Trib2, and Trib3, all of which are serine-threonine kinase-like molecules characterized by a central serine/threonine kinase-like domain and a C-terminal binding site for $\mathrm{E} 3$ ubiquitin ligases (Hegedus et al., 2006). However, these proteins are catalytically inactive owing to the absence of conserved residues of the characteristic adenosine triphosphate binding site and catalytic core motif. Thus, tribbles most likely function as adapter or scaffold proteins. It has been shown that tribbles participate in a range of cellular processes such as in the regulation of MAPK signaling, apoptosis, and cell cycle progression (Mata et al., 2000; Seher and Leptin, 2000). Several studies also indicated that tribbles may function as both oncogenes and tumor suppressors in various cancers. For example, Trib1 could negatively regulate proliferation in vascular smooth muscle cells through blocking of MKK4/JNK-induced proliferation (Sung et al., 2007). On the other hand, it could accelerate the onset of HoxA9/Meis1-induced acute myeloid leukemia via enhancing ERK-induced inhibition of apoptosis (Jin et al., 2007). Studies also demonstrated that high levels of Trib1 expression are associated with human hematopoietic malignancies (Dedhia et al., 2010). There has been no report investigating the roles that TRIB1 play in the development of pancreatic cancer, although it is known to express at a high level in the pancreas (Su et al., 2002).

Therefore, we conducted this case-control study to evaluate the association. In the allele analysis, we found that the rs 2980879 polymorphism of the TRIB1 gene was associated with pancreatic cancer. In addition, the genotype analysis of this SNP showed a significant association. The other two polymorphic sites showed no significant association with this disease. Furthermore, we analyzed the haplotype and linkage disequilibrium of these 3 sites, and the results showed no special indications. 
In conclusion, our study indicated that the TRIB1 gene, especially the rs 2980879 polymorphism, is related to the development of pancreatic cancer in the Chinese Han population.

\section{Conflicts of interest}

The authors declare no conflict of interest.

\section{REFERENCES}

Amundadottir L, Kraft P, Stolzenberg-Solomon RZ, Fuchs CS, et al. (2009). Genome-wide association study identifies variants in the $\mathrm{ABO}$ locus associated with susceptibility to pancreatic cancer. Nat. Genet. 41: 986-990.

Bardeesy N and DePinho RA (2002). Pancreatic cancer biology and genetics. Nat. Rev. Cancer 2: 897-909.

Dedhia PH, Keeshan K, Uljon S, Xu L, et al. (2010). Differential ability of Tribbles family members to promote degradation of C/EBPalpha and induce acute myelogenous leukemia. Blood 116: 1321-1328.

Hegedus Z, Czibula A and Kiss-Toth E (2006). Tribbles: novel regulators of cell function; evolutionary aspects. Cell Mol. Life Sci. 63: 1632-1641.

Jin G, Yamazaki Y, Takuwa M, Takahara T, et al. (2007). Trib1 and Evil cooperate with Hoxa and Meis1 in myeloid leukemogenesis. Blood 109: 3998-4005.

Kathiresan S, Melander O, Guiducci C, Surti A, et al. (2008). Six new loci associated with blood low-density lipoprotein cholesterol, high-density lipoprotein cholesterol or triglycerides in humans. Nat. Genet. 40: 189-197.

Kiss-Toth E, Bagstaff SM, Sung HY, Jozsa V, et al. (2004). Human tribbles, a protein family controlling mitogen-activated protein kinase cascades. J. Biol. Chem. 279: 42703-42708.

Li D, Xie K, Wolff R and Abbruzzese JL (2004). Pancreatic cancer. Lancet 363: 1049-1057.

Liu J, Li ZQ, Li JY, Li T, et al. (2012). Polymorphisms and haplotypes in the YWHAE gene increase susceptibility to bipolar disorder in Chinese Han population. J. Clin. Psychiatry 73: e1276-e1282.

Lu XH, Wang L, Li H, Qian JM, et al. (2006). Establishment of risk model for pancreatic cancer in Chinese Han population. World J. Gastroenterol. 12: 2229-2234.

Malats N (2001). Gene-environment interactions in pancreatic cancer. Pancreatology 1: 472-476.

Mata J, Curado S, Ephrussi A and Rorth P (2000). Tribbles coordinates mitosis and morphogenesis in Drosophila by regulating string/CDC25 proteolysis. Cell 101: 511-522.

Pandey A, Forte V, Abdallah M, Alickaj A, et al. (2011). Diabetes mellitus and the risk of cancer. Minerva Endocrinol. 36: $187-209$.

Petersen GM, Amundadottir L, Fuchs CS, Kraft P, et al. (2010). A genome-wide association study identifies pancreatic cancer susceptibility loci on chromosomes 13q22.1, 1q32.1 and 5p15.33. Nat. Genet. 42: 224-228.

Qiu D, Kurosawa M, Lin Y, Inaba Y, et al. (2005). Overview of the epidemiology of pancreatic cancer focusing on the JACC Study. J. Epidemiol. 15 (Suppl 2): S157-S167.

Seher TC and Leptin M (2000). Tribbles, a cell-cycle brake that coordinates proliferation and morphogenesis during Drosophila gastrulation. Curr. Biol. 10: 623-629.

Shi YY and He L (2005). SHEsis, a powerful software platform for analyses of linkage disequilibrium, haplotype construction, and genetic association at polymorphism loci. Cell Res. 15: 97-98.

Siegel R, Naishadham D and Jemal A (2012). Cancer statistics, 2012. CA Cancer J. Clin. 62: 10-29.

Su AI, Cooke MP, Ching KA, Hakak Y, et al. (2002). Large-scale analysis of the human and mouse transcriptomes. Proc. Natl. Acad. Sci. U. S. A. 99: 4465-4470.

Sung HY, Guan H, Czibula A, King AR, et al. (2007). Human tribbles-1 controls proliferation and chemotaxis of smooth muscle cells via MAPK signaling pathways. J. Biol. Chem. 282: 18379-18387.

Verma M (2005). Pancreatic cancer epidemiology. Technol. Cancer Res. Treat. 4: 295-301.

Willer CJ, Sanna S, Jackson AU, Scuteri A, et al. (2008). Newly identified loci that influence lipid concentrations and risk of coronary artery disease. Nat. Genet. 40: 161-169.

Wu C, Miao X, Huang L, Che X, et al. (2012). Genome-wide association study identifies five loci associated with susceptibility to pancreatic cancer in Chinese populations. Nat. Genet. 44: 62-66.

Yokoyama T and Nakamura T (2011). Tribbles in disease: Signaling pathways important for cellular function and neoplastic transformation. Cancer Sci. 102: 1115-1122. 